\title{
A Smart Poultice with Reconfigurable Sensor Array for Wearable Cardiac Healthcare
}

\author{
Long Yan \\ Dept. of Electrical Engineering \\ KAIST \\ Daejeon, Republic of Korea \\ yanlong@eeinfo.kaist.ac.kr
}

\author{
Hoi-Jun Yoo \\ Dept. of Electrical Engineering \\ KAIST \\ Daejeon, Republic of Korea \\ hjyoo@ee.kaist.ac.kr
}

\begin{abstract}
A smart poultice with reconfigurable sensor array is implemented by using planar fashionable circuit board (P-FCB) technology for wearable cardiac healthcare. It contains 1) 25electrode array either for vital signal sensing or for external data communication, 2) a thin flexible battery, 3) a fabric inductor, and 4) a fabric circuit board on which a low power silicon chip is directly integrated. Start/stop operation of the smart poultice is realized by inductive-coupled remote controller with $8 \mathrm{~b}$ ID verification function and external low power data transaction is achieved by using duty cycled body channel communication.
\end{abstract}

Keywords- Wearable cardiac healthcare; reconfigurable sensing; thoracic impedance variance (TIV); remotely controlled system start-up; planar fashionable circuit board (P-FCB);

\section{INTRODUCTION}

Irregular life style such as lack of physical exercise, overeating, and excessive drinking leads to a heart disease. Actually, it is the worldwide largest killer and claims over 10 millions of lives a year. Among them, failure of the proper blood circulation by a blockage due to a build-up of fatty deposits accumulated on the inner walls of blood vessels dominantly occurs. Since it is a chronic process, the heart attack may be the first warning. In such circumstance, a noninvasive preventive measure of exact pumping capacity of the heart and abnormal patterns of the blood flow is essential. However, wearable ECG monitoring [1] provides insufficient information because detailed expansion and contraction of the blood vessel require monitoring the heart of mechanics rather than the heart of electricity.

In this work, we propose a smart poultice that can measure thoracic impedance variance (TIV) [2] from the change of aortic blood volume and velocity change at each cardiac cycle. Combined with ECG signals from multi points at the thorax, it enables a preemptive medicine by noninvasively providing important hemodynamic information such cardiac output and heart rate in daily life. A customized system on chip (SoC) is integrated on a 4-layer fabric circuit board with a flexible battery as a poultice-like plaster. It can reconfigure 25electrode array and optimize them in-situ to automatically consider the user dependency of the TIV/ECG signals. Benefits from the proposed smart poultice, patient who suffers from

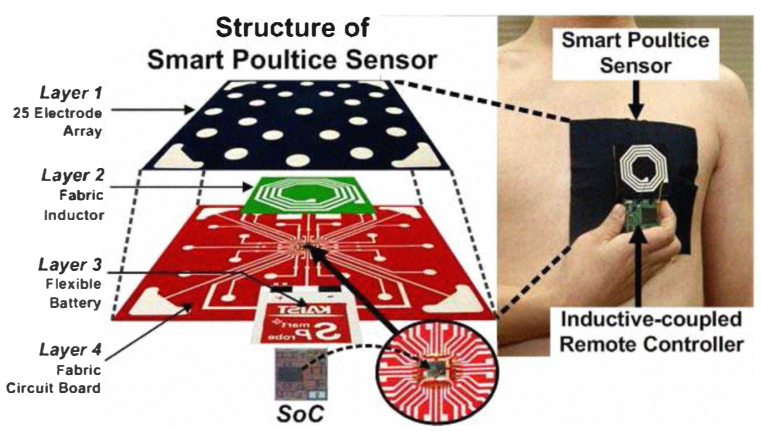

Figure 1. Wearable cardiac healthcare system with proposed smart poultice sensor

hypertension or heart failure can take prophylactic measurement on abnormal symptoms of the heart and even give the patient or doctor warning to avoid an unexpected accident.

\section{SYSTEM ARCHITECURE AND ITS OPERATION}

\section{A. Architecture}

Figurel shows the structure of the wearable cardiac healthcare system with the proposed smart poultice sensor. A $15 \mathrm{~cm} \times 15 \mathrm{~cm}$ 4-layer patch fabricated by P-FCB [3] consists of Layer-1, 25-electrode array for reconfigurable TIV/ECG sensing, Layer-2, fabric inductor $(2.2 \mu \mathrm{H}, \mathrm{Q}=9.6)$ for remote system start-up, Layer-3, thin flexible battery $(1.5 \mathrm{~V}, 30 \mathrm{mAh})$, and Layer-4, fabric circuit board on which a $5 \mathrm{~mm} \times 5 \mathrm{~mm}$ SoC [4] is directly wire bonded and plastic molded for the protection. Layer-1 has 1)16 voltage sensing electrodes divided into 4 sections, 2) 2 anodes and 2 cathodes electrodes for current injection and 3) 4 ground electrodes and 1 reference electrode. A user puts the patch on the chest to monitor the TIV in 8 different configurations and 8-point of ECGs with time multiplexed operation. Figure 2 shows the overall block diagram of the proposed $\mathrm{SoC}$ architecture. It consists of 1) a system start-up module for remote battery control, 2) reconfigurable $4 \times 4$-electrode sensor front ends for voltage sensing and digitization of TIV/ECG, 3) a differential sinusoidal current generator, which is connected to 2 pairs current electrodes, for TIV measurement, 4) a digital module

Permission to make digital or hard copies of all or part of this work for personal or classroom use is granted without fee provided that copies are not made or distributed for profit or commercial advantage and that copies bear this notice and the full citation on the first page. To copy otherwise, to republish, to post on servers or to redistribute to lists, requires prior specific permission and/or a fee. PERVASIVEHEALTH 2010, March 22-25, Munchen, Germany Copyright (C) 2010 ICST 978-963-9799-89-9 DOI 10.4108/ICST.PERVASIVEHEALTH2010.8871 


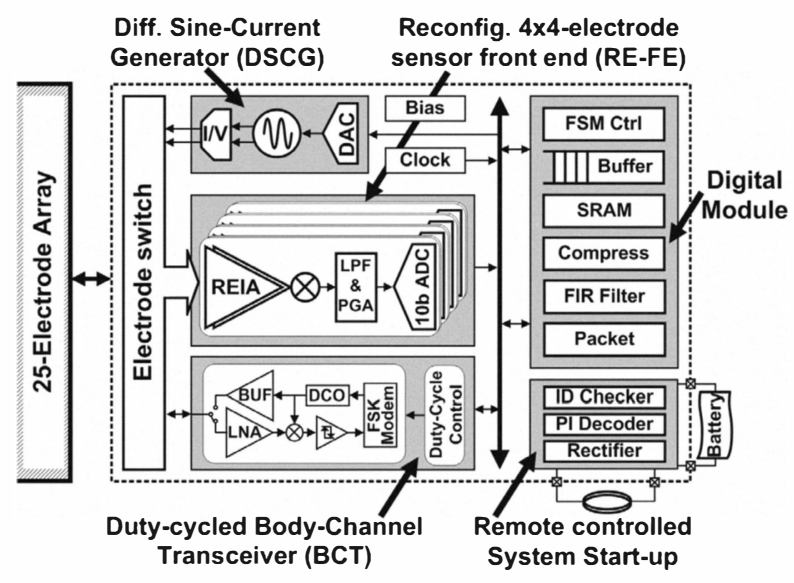

Figure 2. Proposed architecure of smart poultice IC

containing FSM controller with special purpose registers (SPR), a $20 \mathrm{kB}$ data storage, a $10^{\text {th }}$ order FIR filter, an 8:1 data compression block, and a packet encoder/decoder, and 5) a low power duty-cycled body channel transceiver (BCT) in Figure 2 for external data communication and sensor parameter modification through human body.

\section{B. Operation}

User can start the system by inductively coupled power switch with ID verification. An 8b ID which is amplitude shift keying (ASK) modulated in $13.56 \mathrm{MHz}$ with $4 \mathrm{~b}$ pulse interval encoding is provided by remote controller in Figure.1. Then the system start-up module rectifies the incoming ID packet and asynchronously demodulates and authenticates the ID with on-chip preprogrammed $8 \mathrm{~b}$ code for the flexible battery to turn on the $\mathrm{SoC}$ as shown in Figure 3 (b). 8-ponit of ECGs can be measured by 8 electrodes which is connected to 2 of 4 sensor readout front ends in time multiplexed function while TIV can be monitored by the other 8 voltage sensing electrodes differentially. In order to find the optimum sensing points automatically, it scans 8 different electrodes in diagonal sections for $8 \mathrm{~s}$ using the same current injection electrodes. Then the current injection electrodes are changed from anode0-cathode0 to anodel-cathodel with mode swapping between ECG mode and TIV mode. The BCT is activated every $4 \mathrm{~s}$ to listen from the base station for $0.1 \mathrm{~s}$, and it updates channel assessments with new system command. When the $20 \mathrm{kB}$ storage is full and the communication channel is clean, the stored data is transmitted through BCT to the base station, and then system resumes the sensing operation.

\section{IMPELEMENTATION AND MEASUREMENTS}

Figure 3 (a) shows the implemented adhesive smart poultice with film paper protection. The adhesive on the electrode array ensures stable contact with the skin during the smart poultice operation. Figure 3 (b) shows the operation of system start-up module with remote battery control through inductive coupling. It can asynchronously decode $8 \mathrm{~b}$ ID code (00100111) from the rectified packet. Figure 4 (c) shows the

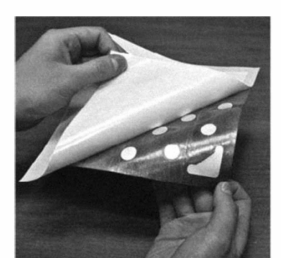

(a)

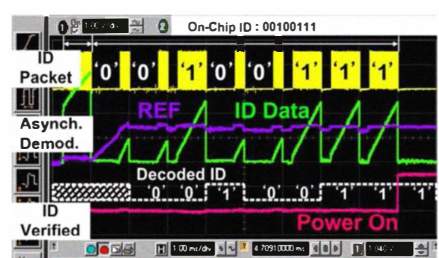

(b)

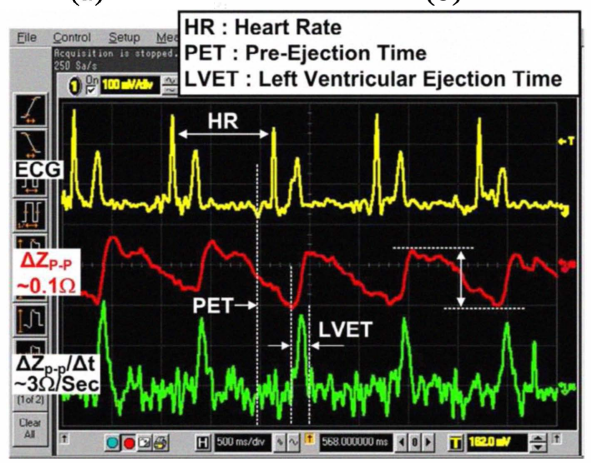

(c)

Figure 3. (a) Implemented adhesive smart poultice, (b) Measurement of remote battery control, (c) Measured waveforms of TIV with ECG

measured waveforms of TIV and ECG signals. A peak-peak TIV of $0.1 \Omega$ is detected with ECG signals while consuming only $2.4 \mathrm{~mW}$ supplied by an embedded thin flexible battery inside the smart poultice. Combined with the heart rate (HR) from ECG signals, left ventricular ejection time (LVET) with pre-ejection time (PET) can be derived so that the cardiac output, which indicates the pumped blood volume from the heart, can be acquired.

\section{CONCLUSION}

A smart poultice is implemented by using P-FCB for wearable cardiac healthcare. It can continuously monitor vital signals over 10hours with single embedded flexible battery and a reconfigurable sensor array. User can remotely control the smart poultice and its recorded data can be transmitted through the human body based on a duty cycled body channel communication technique with peak power consumption of $3.9 \mathrm{~mW}$.

\section{REFERENCES}

[1] J.Yoo, et.al., "A $5.2 \mathrm{~mW}$ Self-Configured Wearable Body Sensor Network Controller and a 12uW 54.9\% Efficiency Wirelessly Powered Sensor for Continuous Health Monitoring System," IEEE JSSC, Vol.45, pp.178-188, Jan. 2009.

[2] A. Nowakowski, et al., "Advances in electrical impedance methods in medical diagnostics," Bulletin of the Polish Academy of Sciences, Tech. Papers, Vol.53, No.3, pp.231-243, 2005

[3] H. Kim, et.al., "A $1.12 \mathrm{~mW}$ continuous healthcare monitor chip integrated on a planar-fashionable circuit board," IEEE ISSCC pp.150151, Feb. 2008

[4] L.Yan, et.al., "a $3.9 \mathrm{~mW}$ 25-Electrode Reconfigured Thoracic Impedance/ECG SoC with Body-Channel Transponder," IEEE ISSCC pp.490-491, Feb. 2009. 